\title{
Quasi-Lumped Element Implementation of a Wide-Band Dual-Band Filter Based on Reactance Transformations
}

\author{
Leokadia Ndeapo Popyeni NEPAYA, Riana Helena GESCHKE \\ Dept. of Electrical Engineering, University of Cape Town, Rondebosch, 7700, South Africa \\ lpnepaya@unam.na,riana.geschke@uct.ac.za
}

Submitted November 29, 2018 / Accepted April 30, 2019

\begin{abstract}
The synthesis and design of a quasi-lumped element dual-band filter is presented for a radiometry application. Band 1 is from 0.45 to $0.86 \mathrm{GHz}$ and band 2 from 1.24 to $1.75 \mathrm{GHz}$. The filter circuit model has no theoretical bandwidth limitation and is implemented on multilayer substrate. Controlled suppression between the two bands is demonstrated. Agreement between simulation and measurement confirms the feasibility of the design method. The effect of dominant parasitic elements in the quasi-lumped element implementations is detailed.
\end{abstract}

\section{Keywords}

Quasi lumped element filters, wide-band filter, dualband filter, reactance transform method

\section{Introduction}

In this paper we present a dual-band filter based on a synthesis method without bandwidth limitation. At the lower microwave frequencies, where the size of the transmission line-based filters is large, quasi lumped element (QLE) filters have a size advantage over distributed filters. QLE filters could be smaller than equivalent distributed filters and can realise filters with large bandwidths. The filters are often implemented on multilayer technologies such as LTCC (low temperature co-fired ceramic) and LCP (Liquid Crystal Polymer) or on CMOS (Complementary Metal Oxide Semiconductor) at frequencies above $60 \mathrm{GHz}$.

A single-band wideband filter design on LCP, based on the cascade of a lowpass and highpass filter, with a similar fractional bandwidth and frequency range as the filters presented in this paper, is described in [1]. While this is a single-band filter, it provides comparative data on element Q-factors for quasi-lumped elements on LCP in a similar frequency range as the filters in this paper.

A single band Chebyshev filter on LCP in [2] is the basis for two passbands. This is achieved by a transformation that replaces a second order LC resonator filter with a fourth order network. The realised filter is compact and easy to implement. Its downside is that the circuit topology provides limited suppression between passbands.

While the size advantage of quasi-lumped elements (QLE) over distributed elements are pronounced at frequencies below $10 \mathrm{GHz}$, it is not limited to the lower $\mathrm{GHz}$ frequencies. QLE are also used at frequencies up to $100 \mathrm{GHz}$. Between 50 and $100 \mathrm{GHz}$, the size of quasi-lumped elements is comparable to distributed elements. An example of filters based on QLE in CMOS technology are found in [3], where single-layer capacitors are preferred to multilayer capacitors, to reduce loss. Inductors at $\mathrm{mm}$-wave frequencies also need special care in design [4].

Recently, a synthesis method to produce exact lumped element models for multi-band filters was developed [5]. The wideband version of this method for multi-band lumped elements filters [6] is based on rational transformation functions for frequency mapping. It transforms each single band lumped element into a one-port cascaded circuit of inductors and capacitors, according to the required number of passbands. The lumped element circuit model of this paper is derived from this procedure. One key difference between our work and previous work, for example [2], is that our circuit model is based on an exact synthesis method which is equally applicable for narrowband and wideband multiband filters. The lowpass lumped element filter and the dual-band lumped-element filter models are shown in Fig. 1.

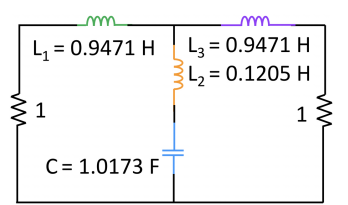

(a)

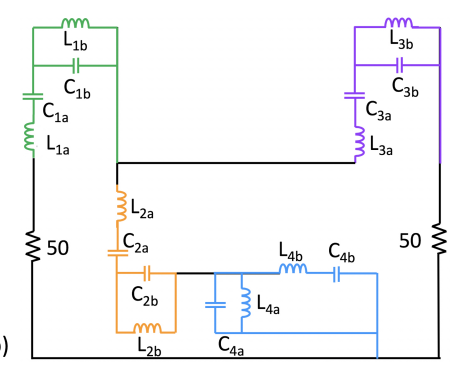

Fig. 1. A dual-band filter results (b) after a transformation from the basis lowpass filter in (a). Each inductor and capacitor in the lowpass filter is transformed into its corresponding equivalent reactance for a dual-band filter as indicated by the same colours. 


\section{Synthesis Based on a Wide-Band Reactance Transform}

A lowpass prototype filter for an elliptic functions is selected for the finite-frequency transmission zeros realization. A lowpass prototype is shown in Fig. 1(a). The low pass filter shunt branch with the series resonant L-C circuit realizes a finite frequency transmission zero above the pass band. The element values for an elliptic low pass prototype filter are obtained for order 3 from [7] where $g_{1}=L_{1}, g_{2}=C, g_{2}^{\prime}=L_{2}$ and $g_{3}=L_{3}$. Order 3 is a lowest order that results into a low number of elements in a dual band filter (in this case eight capacitors and eight inductors) while attaining finite transmission zeros. The low pass prototype is transformed to a multi-band network in Fig. 1(b). Each element in Fig. 1(a) represents four elements in a dual band filter Fig. 1(b) as indicated by the matching colour.

The dual-band filter frequency specifications extends from $0.45 \mathrm{GHz}$ to $0.86 \mathrm{GHz}$ for band 1 and from $1.24 \mathrm{GHz}$ to $1.75 \mathrm{GHz}$ for band 2 . The synthesis process begins with mapping the real pass band edge frequencies to the prototype frequency domain using the general mapping function relationship defined in [5].

$$
\Omega=\frac{\omega}{\omega_{U_{N}}} .
$$
follows:

The prototype pass band edge frequencies are found as

Band 1:

$$
\Omega=(0.2571,0.4937) .
$$

Band 2:

$$
\Omega=(0.7057,1.000)
$$

The prototype pass band specifications obtained with (1), where $\omega_{U_{N}}$ denote the high band-edge of the highest pass-band and is equal to $1.75 \mathrm{GHz}$. It carries $2 \mathrm{~N}$ pass bands resulting in a set of $4 N$ pass band edge frequency in the actual frequency domain presented in (4) where $N=2$ for a dual band filter.

$$
\begin{aligned}
\Omega_{i}, \Omega_{i}^{\prime} & =(-1.0000,-1.0000) ;(-0.7057,1.0000) ; \\
& (-0.4937,-1.0000) ;(-0.2571,1.0000) ; \\
& (0.2571,-1.0000) ;(0.4937,1.0000) ; \\
& (0.7057,-1.0000) ;(1.0000,1.0000)
\end{aligned}
$$

The set of coordinates in( 4) are plotted in Fig. 2. The coordinate set in (4) is applied to (5) to obtain a set of $4 \mathrm{~N}$ linear equations in terms of the unknown coefficients.

$$
\rho_{2}\left(\Omega_{i}\right)-q_{2}\left(\Omega_{i}\right) \Omega^{\prime}\left(\Omega_{i}\right)=0
$$

where $\rho_{2}\left(\Omega_{i}\right)=\alpha_{2} N \Omega_{i} 2 N+\alpha_{2} N-1 \Omega_{i} 2 N-1+\cdots+\alpha_{1} \Omega_{i}+1$ and $q_{2}\left(\Omega_{i}\right) \Omega^{\prime}\left(\Omega_{i}\right)=\beta_{2} N-1 \Omega_{i} 2 N-1+\beta_{2} N-2 \Omega_{i} 2 N-2+$ $\cdots+\beta_{1} \Omega_{i}+\beta_{0}$. For search of values of unknown coefficients $\alpha_{i}$ and $\beta_{i}$.
The expression in (5) is then presented in matrix notation as $\mathbf{A X}=\mathbf{B}$. The matrix $\mathbf{A}$, eight rows of which $(i=8)$ are in form $\left(\Omega_{i} 2 N, \Omega_{i} 2 N-1, \Omega_{i} 2 N, \cdots, \Omega_{i}, \Omega_{2} \Omega_{i} 2 N-\right.$ $\left.1, \cdots, \Omega \Omega_{i}, 1\right)$ and is detailed in Tab. 1 .

The matrix $\mathbf{X}$ consists of unknown coefficients in the sequence of $\left.\alpha_{2} N, \alpha_{2} N-1, \cdots, \alpha_{1},-\beta_{2} N-1, \cdots,-\beta, \beta_{0}\right)$. In the result of calculation, the matrix $\mathbf{B}$ and $\mathbf{X}$ are created as follows:

$$
\mathbf{B}=\left(\begin{array}{l}
-1.0000 \\
-1.0000 \\
-1.0000 \\
-1.0000 \\
-1.0000 \\
-1.0000 \\
-1.0000 \\
-1.0000
\end{array}\right)
$$

$$
\mathbf{X}=\left(\begin{array}{c}
11.1615 \\
0.0000 \\
-8.5167 \\
-0.0000 \\
5.9251 \\
0.0000 \\
-2.2804 \\
0.0000
\end{array}\right)
$$

The rational mapping function $\Omega^{\prime}=\rho_{2}(\Omega) / q_{2}(\Omega)$ as a function of $\Omega$, found according to the method presented in [5], [6] is:

$$
\Omega^{\prime}=\frac{11.1615 \Omega^{4}-8.5167 \Omega^{2}+1}{5.9251 \Omega^{3}-2.2804 \Omega} .
$$

The results of calculation by (6) are shown in Fig. 2.

A driving point function can be indicated in the Foster topology by indicating the function as a sum of partial fractions. The general driving point function, (6), for this dual-band filter case as a Foster expansion according to the method in [5] is:

$$
H(S)=\frac{0.4385}{S}+\frac{0.1369}{S-\mathrm{j} 0.6204}+\frac{0.1369^{*}}{S+\mathrm{j} 0.6204}+1.8837 S
$$

while the Mixed Cauer expansion results from evaluating one pole at zero and one pole at infinity inside the continued fraction expansion. Two coefficients are computed between inversions as:

$$
H(S)=1.8837 S+\frac{0.4385}{S}+\frac{1}{3.6513 S+\frac{1.4053}{S}} .
$$

Both expansions narrate a circuit in terms of impedance function in which elements are connected in series or an admittance function where the elements are connected in parallel. Both expansions lead to the same circuit elements for the specific case of a dual-band filter. 


\begin{tabular}{|c|c|c|c|c|c|c|c|}
\hline 1.000 & -1.0000 & 1.0000 & -1.0000 & -1.0000 & 1.0000 & -1.0000 & 1.0000 \\
\hline 0.2480 & -0.3515 & 0.4980 & -0.7057 & 0.3515 & -0.4980 & 0.7057 & -1.0000 \\
\hline 0.0594 & -0.1203 & 0.2438 & -0.4937 & -0.1203 & 0.2438 & -0.4937 & 1.0000 \\
\hline 0.0044 & -0.0170 & 0.0661 & -0.2571 & 0.0170 & -0.0661 & 0.2571 & -1.0000 \\
\hline 0.0044 & 0.0170 & 0.0661 & 0.2571 & 0.0170 & 0.0661 & 0.2571 & 1.0000 \\
\hline 0.0594 & 0.1203 & 0.2438 & 0.4937 & -0.1203 & -0.2438 & -0.4937 & -1.0000 \\
\hline 0.2480 & 0.3515 & 0.4980 & 0.7057 & 0.3515 & 0.4980 & 0.7057 & 1.0000 \\
\hline 1.0000 & 1.0000 & 1.0000 & 1.0000 & -1.0000 & -1.0000 & -1.00004 & -1.0000 \\
\hline
\end{tabular}

Tab. 1. Elements of matrix A.

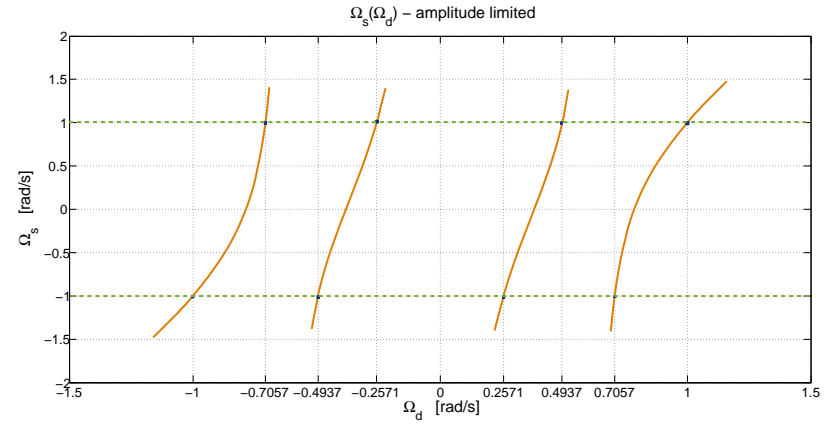

Fig. 2. The wide-band mapping function provides filter symmetry around zero frequency and maps the single band response to a dual-band response.

\begin{tabular}{|c|c|c|c|}
\hline $\mathbf{L}$ & $\mathbf{n H}$ & $\mathbf{C}$ & $\mathbf{p F}$ \\
\hline$L_{1 \mathrm{a}}$ & 3.064 & $C_{1 \mathrm{a}}$ & 4.38 \\
\hline$L_{1 \mathrm{~b}}$ & 8.113 & $C_{1 \mathrm{~b}}$ & 7.014 \\
\hline$L_{2 \mathrm{a}}$ & 1.032 & $C_{2 \mathrm{a}}$ & 34.42 \\
\hline$L_{2 \mathrm{~b}}$ & 0.3899 & $C_{2 \mathrm{~b}}$ & 55.13 \\
\hline$L_{3 \mathrm{a}}$ & 3.064 & $C_{3 \mathrm{a}}$ & 4.38 \\
\hline$L_{3 \mathrm{~b}}$ & 8.113 & $C_{3 \mathrm{~b}}$ & 7.014 \\
\hline$L_{4 \mathrm{a}}$ & 10.24 & $C_{4 \mathrm{a}}$ & 3.471 \\
\hline
\end{tabular}

Tab. 2. Element values of dual band filter in Fig. 1(b).

The transformation replaces each inductor and capacitor in the lowpass circuit by a one port network. Each of the coloured elements of Fig. 1(a) are transformed to equivalent lumped element circuits for a specified dual-band filter in Fig. 1(b). Practically, this means that in the dual-band case each series inductor in Fig. 1(a) is transformed into a series $\mathrm{L}$ and $\mathrm{C}$ plus a parallel L-C combination, as indicated in Fig. 1(b). Since the multi band reactance transformation directly transforms a lowpass filter prototype into a multi-band filter, the implication is that the low pass response is repeated for each pass band. It is clear that the low pass response is repeated as two pass bands with the same shape but different, specified bandwidths. The element values for the dual band filter of Fig. 1(b) is tabulated in Tab. 2 and the theoretical low pass and dual-band responses are shown in Fig. 3.

\section{Practical Design Implementation}

\subsection{Physical Design Layout}

The model in Fig. 1(b) was implemented on a multilayer Mercurywave substrate with dielectric constant of 3.5 and loss tangent of 0.004 with three metallization layers. The layer stackup (material type and layer thickness) is shown in Fig. 4(a). Two implementations, labelled A (Foster expansion) and B (Mixed Cauer expansion), were designed. Both filters have the exactly the same elements but the sequence of elements are different, in this special case of a dual-band filter.

The filter is designed to have two passbands, the first from $0.45 \mathrm{GHz}$ to $0.86 \mathrm{GHz}$, and the second from $1.24 \mathrm{GHz}$ to $1.75 \mathrm{GHz}$. The 3D layout of implementation $\mathrm{B}$, the more compact implementation, is shown in Fig. 4(a). Figure 4(b) shows the layer stackup of six layers and the layer thickness in each case. Fig. 4(c) details a 3D view of the inductor construction.

The suppression between bands is set at $30 \mathrm{~dB}$ as is evident in Fig. 3. There are 16 lumped element components, but symmetry reduces the number of unique components to twelve. The range of largest to smallest reactive element for this filter is 42 . Figure 5 shows the manufactured filters.

Four inductor build structures are detailed in Fig. 4(c). Large inductance values are realised by spiral designs (inductors $L_{1 \mathrm{a}}$ and $L_{4 \mathrm{a}}$ ) and have lower Q-factors depending to the number of turns. Smaller inductances are constructed with meandered line, which has low inductance and higher Q-factors, for example the smallest inductor $L_{2 \mathrm{a}}$ in Fig. 4(c).

The frequency responses of the simulated and measured results are in Fig. 6. Figure 6(a) shows the simulated implementation A versus the lumped element model, (b) shows implementation A simulation against measurement, (c) shows simulated implementation B against measurements and (d) shows A versus B measurement results.

The average passband insertion loss is $0.64 \mathrm{~dB}$ for filter $\mathrm{A}$ and $0.9 \mathrm{~dB}$ for filter B. Good agreement for implementation $\mathrm{A}$ and $\mathrm{B}$ are found. This demonstrates that two different designs produce very similar results- indicating the design method is sound and repeatable. Photographs of filter $\mathrm{A}$ and $\mathrm{B}$ are shown in Fig. 5. Version B of the filter is a more compact implementation, aimed at investigating to what extent the parasitic effects would be more influential in such an implementatation.

The physical size(s) of elements both inductors and capacitors including vias were developed following [8]. S parameter of each element were measured to provide accurary of high level while taking into consideration effects of promixity and subtrate thickness. Design B is build and constructed as shown in Fig. 10 and Fig. 11. 

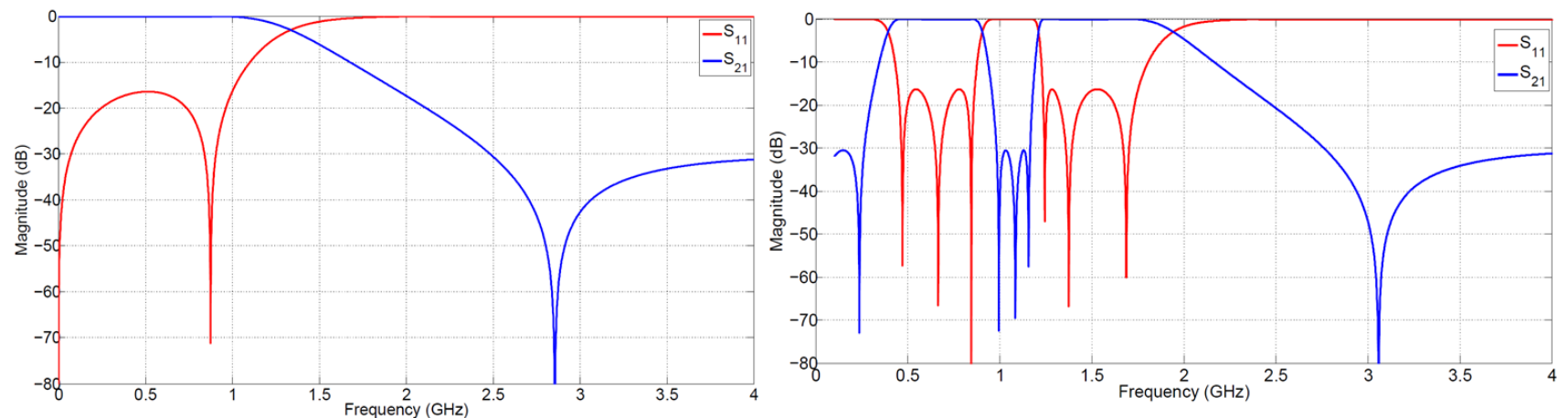

Fig. 3. The theoretical responses of the lowpass and dual-band filter of Fig. 1 on which the practical design is based in this paper. The dual-band filter has finite transmission zeros (in GHz) at zero, 0.238, 0.996,1.085,1.157 and 3.059.

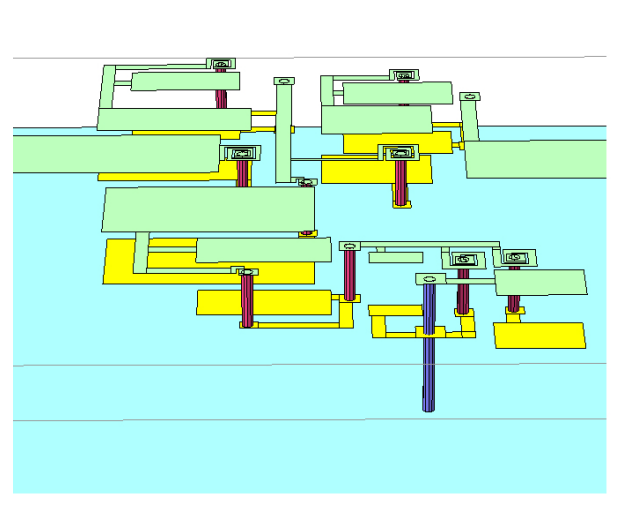

(a)

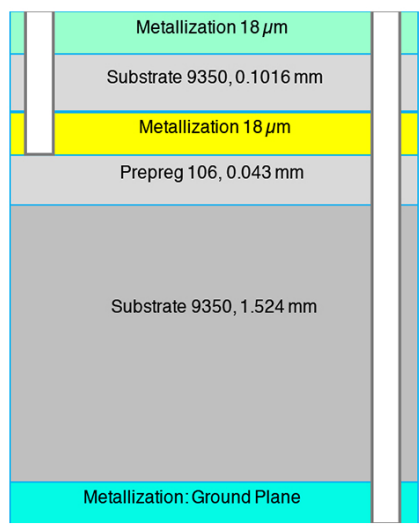

(b)

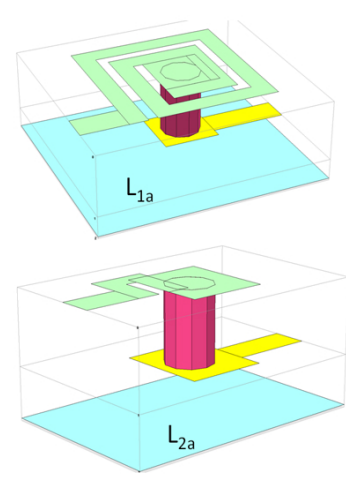

(c)

Fig. 4. (a) 3D view of the designed multilayer filter version B, shown in Fig. 5(b). (b) Layer stackup based on ParkElectrocomp's Mercurywave 9350 and 106 prepreg layers. (c) Inductor implementation for the filter of Fig. 1. The metallization colour scheme of Fig. 4 is used to indicate the use of the metallization layers to build the inductors. The vias are either through vias or blind vias from top to middle metallization layer.
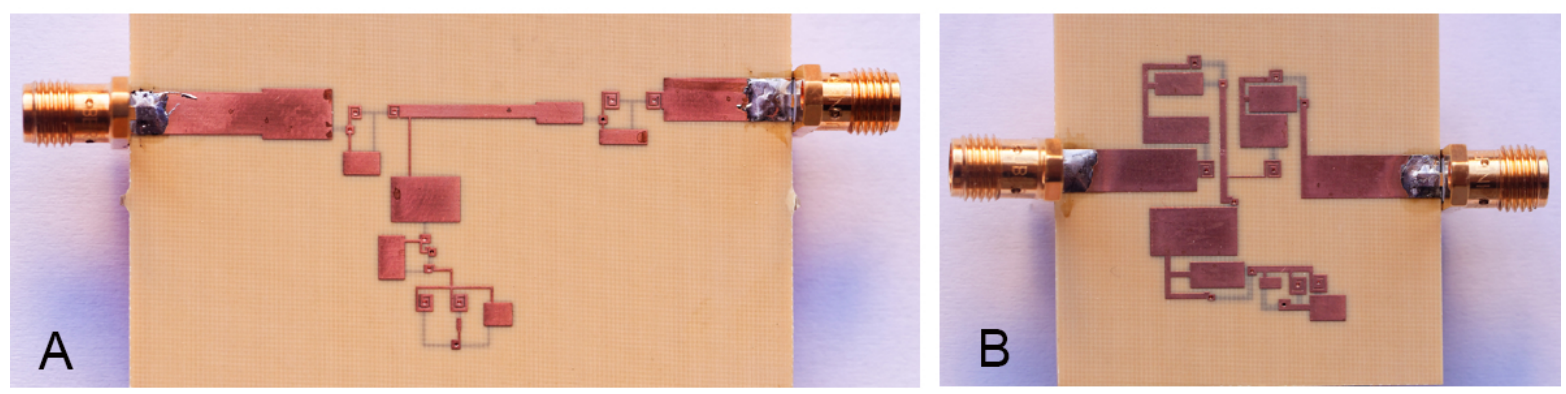

Fig. 5. Implementations A and B of the circuit model in Fig. 1. The elements are the same. Filter B has a different sequence of elements and more compact interconnects. 


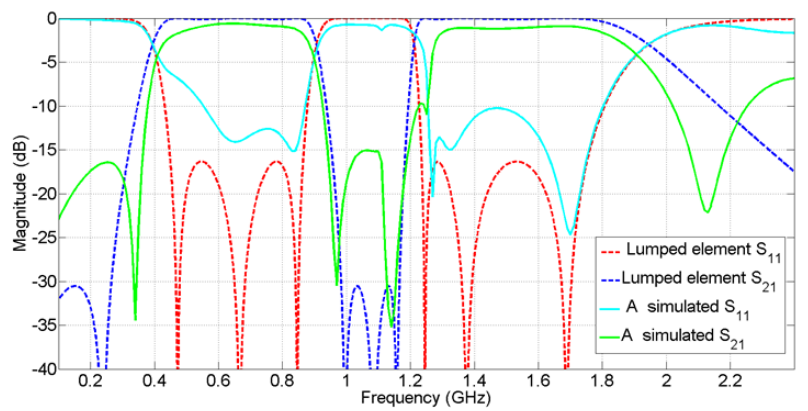

(a)

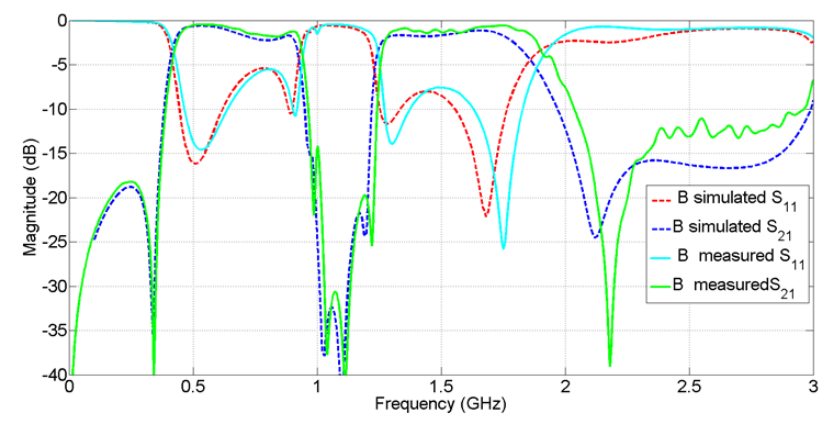

(c)

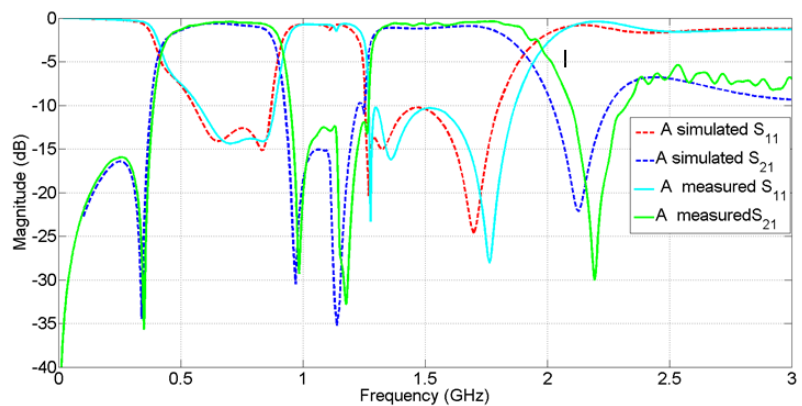

(b)

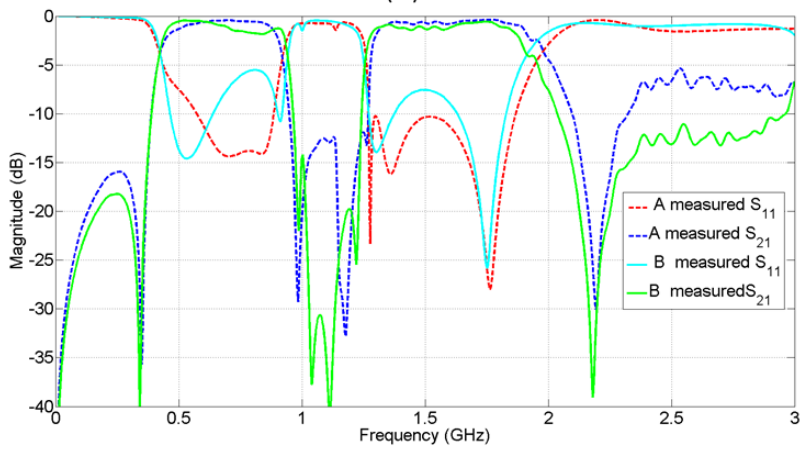

(d)

Fig. 6. S-parameters for (a) Ideal lumped element filter compared to Design A simulated data, (b) Design A simulated against measured data, (c) Design B simulated against measured data and (d) a comparison of design A and design B measured data.

\subsection{The Influence of Non-idealities on the Real- isation of Filters}

For a multi-band filter it is very likely that parasitic elements, in especially the inductor implementation, could influence the realised bandwidths of the filters. In this section, parasitics that may influence the relative bandwidths and filter selectivity are investigated. Parasitic components for inductors are generally more influential than those of the capacitors. The total inductance in each inductor is found from the EM generated equivalent circuit models based on simulation, where each inductor is simulated using Sonnet software to extract the equivalent circuit. The EM simulation includes all effects ranging from substrate properties,layer stackup and dispersion of material properties. Each inductor has the equivalent circuit shown in Fig. 12 . Here, $C_{\mathrm{s}}$ is the fringing capacitance between the inductor turns, $R_{\mathrm{s}}$ the series resistance of the inductor metal, $L_{\mathrm{s}}$ the total inductance, $R_{\mathrm{k}}$ the series resistance of the inductor metal, $L_{\mathrm{k}}$ series inductance of the inductor metal, $C_{\mathrm{ox} 1}$ and $C_{\mathrm{ox} 2}$ the shunt capacitances of the oxide layer, $C_{\text {sub1 }}, C_{\text {sub2 }}$ total shunt capacitance of the dielectric layers and $R_{\text {sub1 }}, R_{\text {sub2 }}$ shunt resistance due to substrate losses.

To analyse the spurious effects on the ideal dual-band circuit of Fig. 1, the EM generated inductor of each inductor in Fig. 4 is inserted in a dual-band model replacing ideal inductor one by one to observe the impact on the frequency. The values of all parasitic elements generated from the four unique inductor designs are detailed in Tab. 3 with inductors in $\mathrm{nH}$, capacitors in $\mathrm{pF}$ and resistance in $\Omega$. Two examples are indicated in Fig. 7 and Fig. 8: in the first the focus is on $L_{1 \mathrm{a}}$, where the generated spurious elements are included in the model of Fig. 7(a) as shown in Fig. 7(b). The dominant spurious effects from inductor $L_{1 \mathrm{a}}$ is the influence on the bandwidth on band two, as is evident from the first graph of Fig. 9, while band one is not affected significantly. In practice inductor $L_{1 \mathrm{a}}$ has a large physical dimension. To model the spurious effects, $L_{1 \mathrm{~b}}$ and $L_{3 \mathrm{~b}}$ in Fig. 8(a) are replaced by the full circuit models including parasitic elements as detailed in Fig. 8(b). The main influence here is the suppression between the pass bands in the second graph of Fig. 9, which if these effects were not included in the design, would have destroyed suppression between the two bands. In the design process these effects were taken into consideration to meet the filter specifications.

\begin{tabular}{|c|c|c|c|c|}
\hline Parasitic elements & $L_{1 \mathrm{a}}$ & $L_{1 \mathrm{~b}}$ & $L_{2 \mathrm{a}}$ & $L_{4 \mathrm{a}}$ \\
\hline$C_{\mathrm{s}}$ & 0.104 & 1.78 & 0.673 & 1.52 \\
\hline$L_{\mathrm{s}}$ & 6.78 & 1.36 & 2.44 & 1.64 \\
\hline$R_{\mathrm{S}}$ & 0.0727 & 0.0323 & 0.0225 & 0.0382 \\
\hline$R_{\mathrm{k}}$ & 0.478 & 51.5 & 5.01 & 47.2 \\
\hline$L_{\mathrm{k}}$ & 0.161 & 0.545 & 0.218 & 0.543 \\
\hline$C_{\mathrm{ox} 1}$ & 0.654 & 0.633 & 0.639 & 0.647 \\
\hline$C_{\mathrm{Ox} 2}$ & 0.672 & 0.641 & 0.641 & 0.654 \\
\hline$R_{\text {sub1 }}$ & 7.68 & 8.75 & 10.4 & 8.51 \\
\hline$R_{\text {sub2 }}$ & 8.08 & 8.11 & 8.58 & 7.89 \\
\hline$C_{\text {sub1 }}$ & 17.1 & 16.7 & 16.9 & 17.0 \\
\hline$C_{\text {sub2 }}$ & 17.7 & 16.4 & 17.0 & 16.6 \\
\hline
\end{tabular}

Tab. 3. Extracted element values of Fig. 12 for each inductor. 

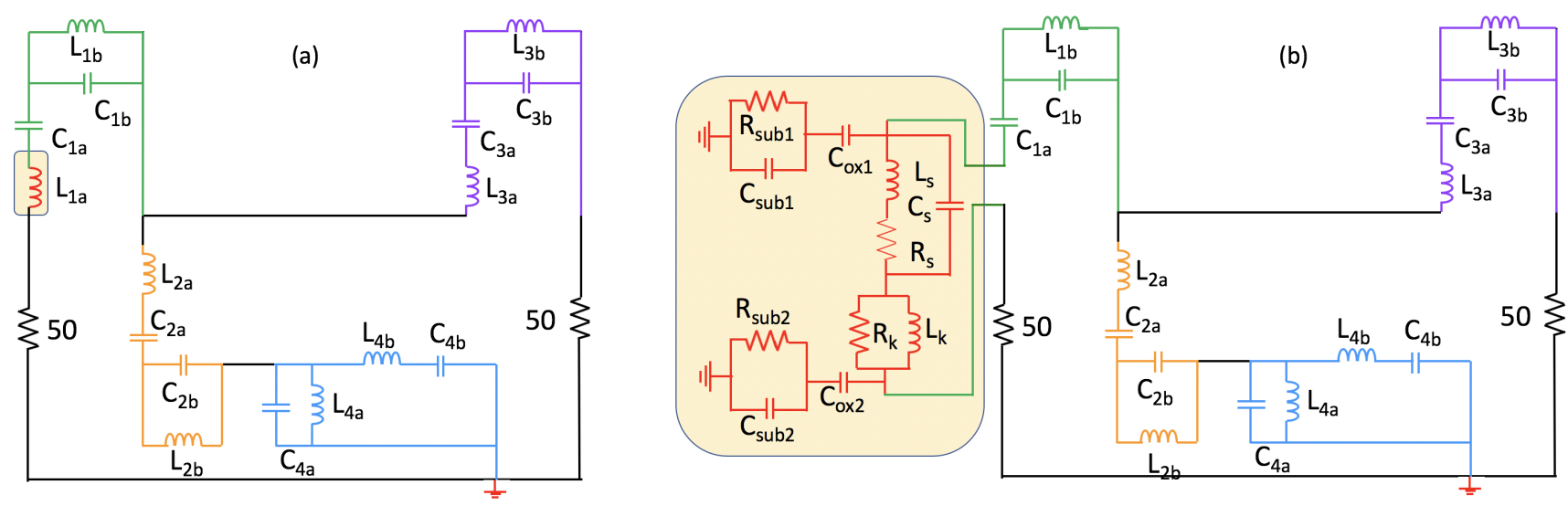

Fig. 7. The ideal circuit model in which the highlighted inductor $L_{1 \mathrm{a}}$ is replaced by the corresponding circuit in (b) with parasitic elements (refer Fig. 12).
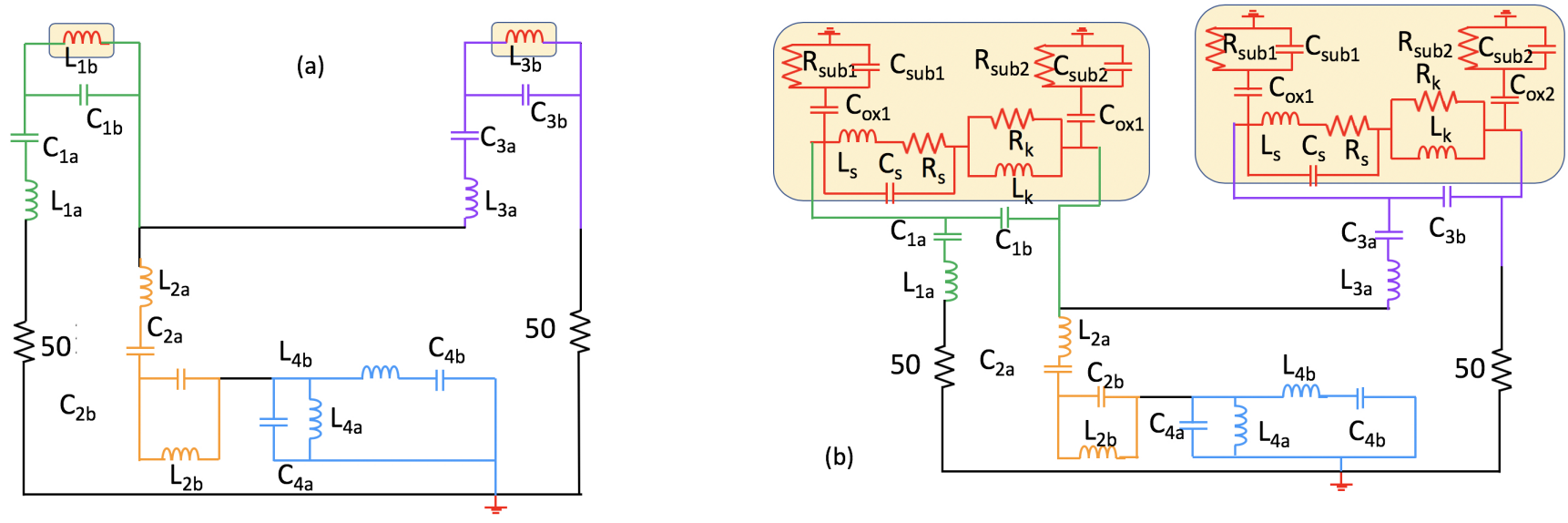

Fig. 8. The ideal circuit model in which the highlighted inductors $L_{1 \mathrm{~b}}$ and $L_{3 \mathrm{~b}}$ in (a) are replaced by their corresponding circuit in (b) with parasitic elements (refer Fig. 12).
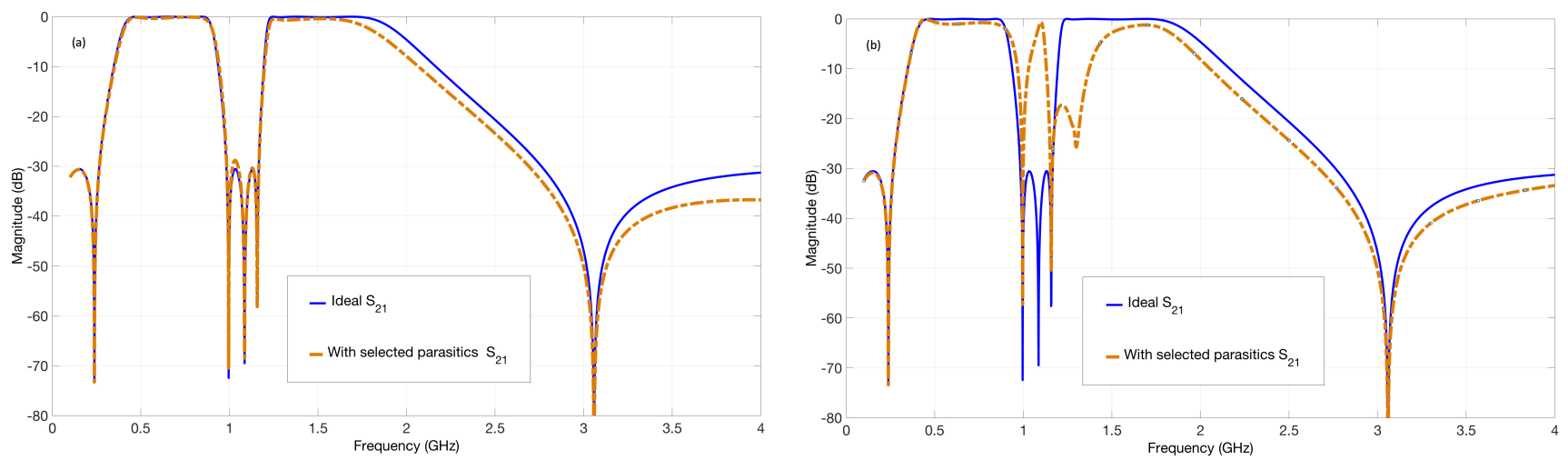

Fig. 9. (a) The effect of the spurious components of the inductor $L_{1 \mathrm{a}}$, detailed in Fig. 7, on the ideal lumped-element filter response. The bandwidth of band two is affected most dramatically. (b) The effect of the spurious components of $L_{1 \mathrm{~b}}$ and $L_{3 \mathrm{a}}$ detailed in Fig. 8, on the lumped element filter response. The suppression between pass bands are affected since the finite transmission zeros between pass bands are shifted, also affecting pass band two bandwidth. 


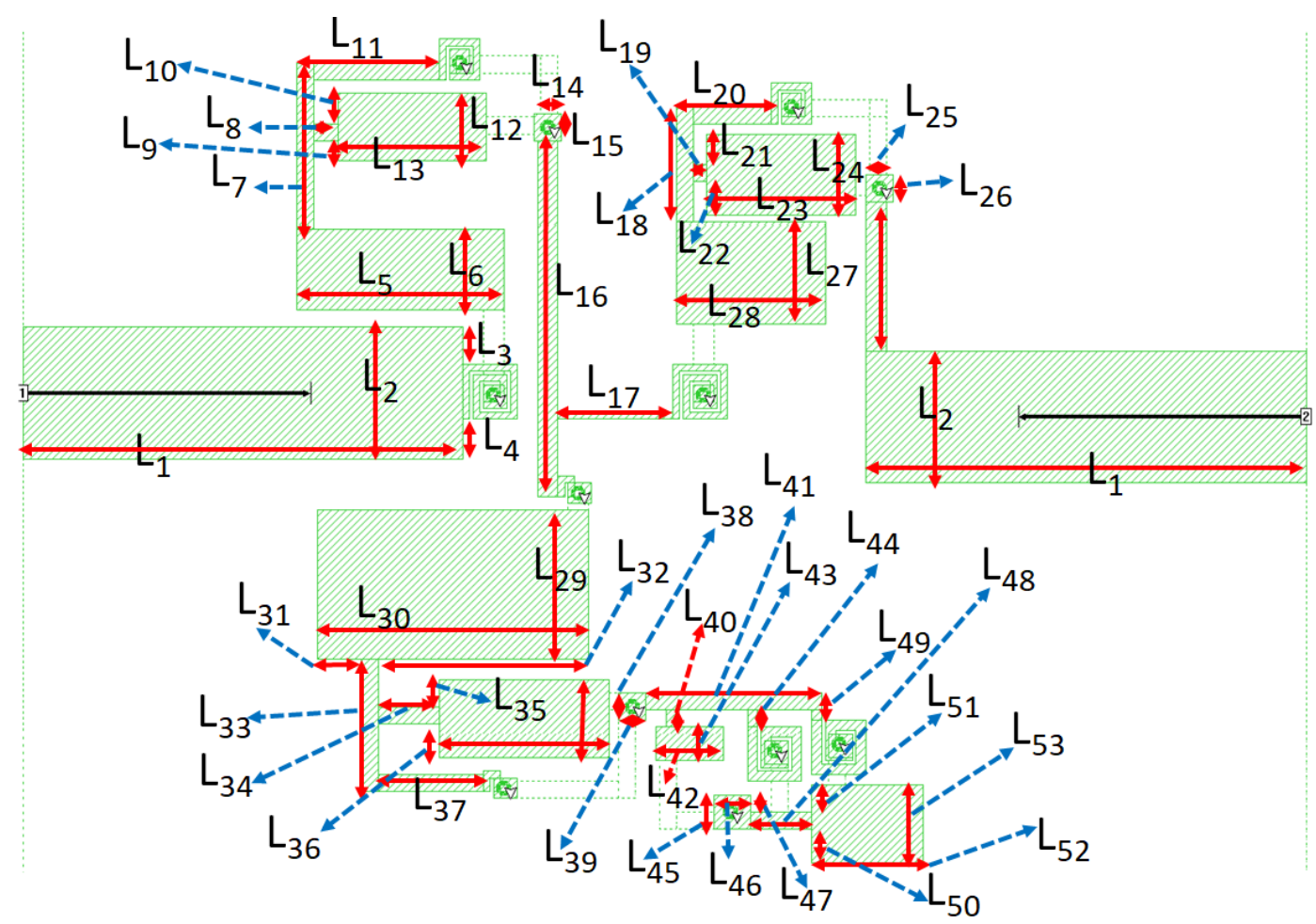

Fig. 10. The lengths of the filter in Fig. 4(a) top layer which is photographed in Fig. 5 labelled B.

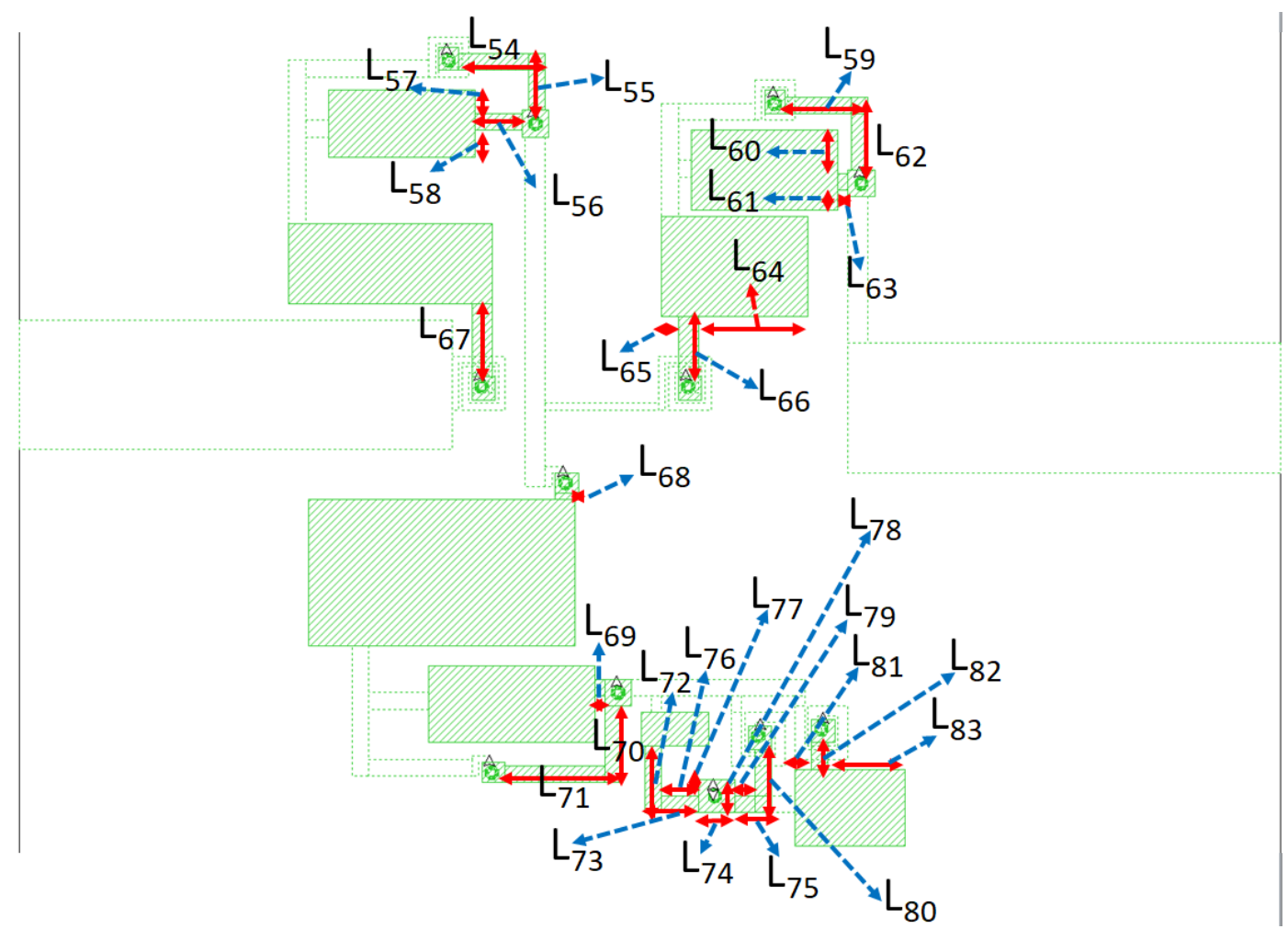

Fig. 11. The lengths of the filter in Fig. 4(a) mid layer which is photographed in Fig. 5 labelled B. 


\begin{tabular}{|c|c|c|c|c|c|c|c|c|}
\hline Filter type & bands & Bands [GHz] & $\mathbf{L}_{\max }[\mathrm{nH}]$ & $\mathbf{L}_{\min }[\mathrm{nH}]$ & $\mathbf{C}_{\max }[\mathrm{pF}]$ & $\mathbf{C}_{\min }[\mathrm{pF}]$ & $\mathbf{L}_{\max } / \mathbf{L}_{\min }$ & $\mathbf{C}_{\max } / \mathbf{C}_{\min }$ \\
\hline Dual band & 2 & band 1: $0.45-0.86$ & & & & & & \\
\hline & & band 2: $1.24-1.75$ & 16.39 & 0.39 & 55.13 & 1.311 & 42.05 & 42.05 \\
\hline Wideband & 1 & 0.45 to 1.75 & 0.26 & 0.015 & 0.28 & 0.12 & 17.51 & 2.23 \\
\hline
\end{tabular}

Tab. 4. Comparison of element value ranges for a wideband and dual-band filter with the same overall bandwidth. This factor is a measure of the challenge level to design a dual-band filter as compared to a single band filter with the same bandwidth.

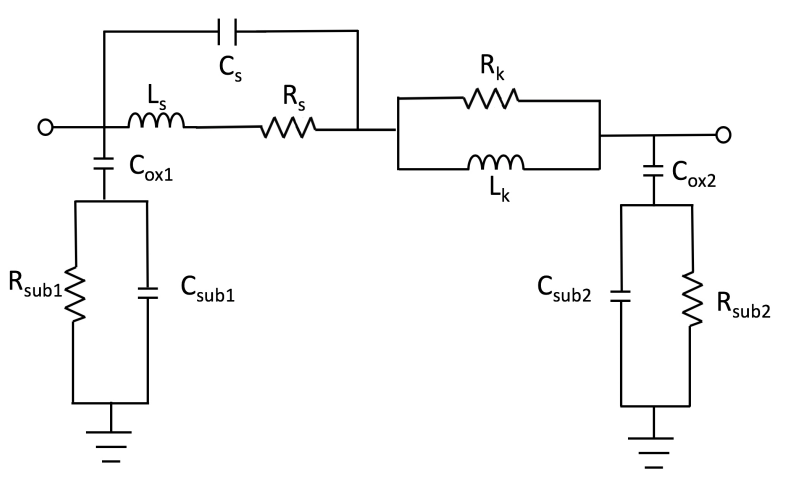

Fig. 12. Equivalent circuit with the parasitic elements for an inductor of the filter of Fig. 1. Here $L_{\mathrm{s}}$ is the desired inductance and the other elements are spurious elements.

\section{Review of Results}

Finally, we compare the spread of reactive element values to a single-band filter with similar overall bandwidth. Table 4 compares a wideband filter (single band) based on the same quasi-elliptic filter of Fig. 1(a) to the multi-band prototype filter of Fig. 1(b). The filter of Fig. 1 has a ratio of maximum to minumum Inductance/Capacitance values of 42, a factor 2.4 higher than a (single) wide band filter with the same overall bandwidth. This confirms a dual-band filter with the same overall bandwidth as a wideband filter is more challenging to design, on account of an increased number of lumped elements, and due to the increased range of element values. Based on our measurement results we demonstrated that this range of lumped element values can be readily implemented on a multi layer stackup.

\section{Conclusion}

This paper demonstrates the use of a reactance transform method to design quasi-lumped element dual-band filters with a centre frequency ratio of 2.3 between passband centre frequencies. The individual fractional bandwidths are $68 \%$ (band 1) and 34\% (band 2). Two implementations with similar results give confidence that the design procedure could be implemented to meet any similar dual-band filter specifications.

\section{Acknowledgments}

The use of Sonnet and NI AWR Microwave Office academic licenses is acknowledged. This work is supported in part by the National Research Foundation of South Africa (Grant number 88100).

\section{References}

[1] QIAN, S., HONG, J. Miniature quasi-lumped-element wideband bandpass filter at $0.5-2-\mathrm{GHz}$ band using multilayer liquid crystal polymer technology. IEEE Transactions on Microwave Theory and Techniques, 2012, vol. 60, no. 9, p. 2799-2807. DOI: 10.1109/TMTT.2012.2205939

[2] BAVIS, A., SWAMINATHAN, A., MINA, E. Liquid crystal polymer-based planar lumped component dual-band filters for dual-band WLAN systems. In Proceedings of the IEEE Radio and Wireless Symposium. Long Beach (USA), 2007, p. 539-542. DOI: 10.1109/RWS.2007.351887

[3] VANAKURU, V. N. R., GODAVARTHI, N., CHAKRAVORTY, A. Miniaturized millimeter-wave narrow bandpass filter in $0.18 \mathrm{up} \mu \mathrm{m}$ CMOS technology using spiral inductors and inter digital capacitors. In Proceedings of the IEEE Conference on Signal Processing and Communications (SPCOM). Bangalore (India), 2014, p. 1-4. DOI: 10.1109/SPCOM.2014.6983960

[4] KRAMER, M., DRAGOMIRESCU, D., PLANA, R. Accurate electromagnetic simulation and measurement of millimeter-wave inductors in bulk CMOS technology. In IEEE Topical Meeting on Silicon Monolithic Integrated Circuits in RF Systems (SiRF). New Orleans (USA), 2010, p. 61-64. DOI: 10.1109/SMIC.2010.5422948

[5] BRAND, T. B. Synthesis Methods for Multi-Band Coupled Resonator Filters. Ph.D. dissertation, Stellenbosch University, 2014. Available at: http://hdl.handle.net/10019.1/95910

[6] BRAND, T. B., MEYER, P., GESCHKE, R.H. Designing multiband coupled- resonator filters using reactance transformations. International Journal of RF and Microwave Computer-Aided Engineering, 2015, vol. 25, no. 1, p. 81-92. DOI: 10.1002/mmce.20826

[7] HONG, J.-S., LANCASTER M. J. Microstrip Filters for RF/Microwave Applications. John Wiley \& Sons, 2001. ISBN: 9780470937297

[8] QIAN, S., HAO Z.-C., HONG, J., et al. Design and fabrication of a miniature highpass filter using multilayer LCP technology. In IEEE European Microwave Conference. Manchester (UK), 2011, p. 187-190. DOI: 10.23919/EuMC.2011.6101685

\section{About the Authors...}

Leokadia Ndeapo Popyeni NEPAYA was born in Olukonda, Namibia. She received her M.Sc. from Beihang University. She is currently working towards Ph.D. degree at University of Cape Town. Her research interests include tunable and reconfigurable design techniques for multiband filters.

Riana Helena GESCHKE was born in Stellenbosch, South Africa. She received her Ph.D. degree from Stellenbosch University in 2004. Her research in the field of Applied Electromagnetics includes systems -level design of radar and radio-astronomy receivers, and at device level, new synthesis and design methods for multiband filters and antennas. 